\title{
Adopting a palliative approach to dementia care in the community: a participatory action research
}

\author{
Lay Ling $\underline{T a n}^{1}$, MBBS, MMed, Wei Fern $\underline{N g}^{1}$, MBBs, Alisson Ching Ching $\underline{\operatorname{Sim}}^{1}$, MB BCh BAO, Sahnan Bin $\underline{\text { Rahim }}{ }^{2}$, BSci
}

\section{INTRODUCTION}

Based on the World Alzheimer Report 2015,(1) dementia is a rising public health concern and is expected to affect 132 million individuals worldwide by 2050. It is an incurable and progressive neurodegenerative disorder with long periods of dependency. ${ }^{(2)}$ However, both professional and family caregivers often do not consider dementia to be a terminal or life-limiting illness. ${ }^{(3,4)}$ This would hamper the provision of optimal end-of-life (EOL) care, which is an integral and critical component of dementia palliative care services. ${ }^{(5)}$

A recent epidemiological study reported a $10 \%$ prevalence of dementia in individuals aged $\geq 60$ years in Singapore. ${ }^{(6)}$ Family members are intimately involved in the care of their loved ones with dementia. ${ }^{(7,8)}$ Decisions on care, including Advance Care Planning (ACP) and EOL care, often have to be made by family caregivers on behalf of persons with dementia (PWD) who may lack the necessary mental capacity.

Singapore ranked 18th out of 40 countries on a 'quality of death index' in a report commissioned by the Lien Foundation in 2010.(9) The report compared standards of EOL care across countries and motivated the development of the National Strategy for Palliative Care in Singapore. It highlighted the need for provision of palliative care services to all patients with lifelimiting illnesses and recommended adequate education and training of healthcare professionals to improve the quality of palliative care.

The Community Psychogeriatric Programme (CPGP) was set up to provide community mental health services for early detection and treatment of psychogeriatric disorders in the eastern sector of Singapore. ${ }^{(10)}$ The CPGP comprised clinical services and home visits to older individuals suffering from mental health problems who face difficulty accessing regular mental health services at hospitals or outpatient services. More than half of the home visits in the CPGP involve PWD with moderate to severe dementia. Moreover, specialist palliative services do not accept referrals unless the patients are predicted to die within the next 12 months from evaluation. ${ }^{(11)}$ Although the CPGP team members were aware of these palliative care needs, they lacked training and clinical experience in rendering such care. Thus, the CPGP decided to undertake an investigation into our clinical practice and to explore how strategies could be developed to support a palliative approach in dementia care in the community.

\section{METHODOLOGY}

This study was granted exemption from review by the Centralised Institutional Review Board (CRIB ref 2020/2275). Action research methodology was adopted, as it focuses on facilitating changes in people's practices, and understanding their practices and the conditions under which they practice. ${ }^{(12)}$ The process helped examine taken-for-granted assumptions and habitual actions that constituted clinical practice. ${ }^{(13)}$

Members of the CPGP team, comprising four nurses and two doctors, participated in a recurring helical action cycle that involved preliminary investigation and problem identification, planning, taking action and collecting data, analysis and reflection and re-planning. ${ }^{(14)}$

Four focus group meetings were convened over six months to complete the first action cycle. Each focus group was led by the principal investigator through a qualitative enquiry process based on key problems identified in clinical practice. Meeting notes and focus group audio transcriptions were used to identify themes, and the Atlas Ti version 8 software (ATLAS.ti Scientific Software Development GmbH, Berlin, Germany) was used to code and analyse the data.

\section{MAIN THEMES PERTAINING TO PROBLEM IDENTIFICATION \\ Difficulty conceptualising dementia as a terminal illness}

Some CPGP staff had difficulty viewing dementia as a terminal illness unless dementia was advanced. Staff also encountered challenges in communicating the concept of dementia as a terminal illness to the family caregivers of PWD.

Nursing Staff (NS) 1: "I need to get my head around dementia as a terminal illness. It is not as hard to explain to the patient when they are stricken with end-stage cancer that they are dying. For dementia, it is difficult to see that they are terminal and dying, particularly if they are still early in the dementia severity."

\section{What constitutes a palliative care approach? \\ Members agreed that a palliative care approach should be adopted when the diagnosis of dementia is established. However, they were unable to elaborate on what constituted a palliative care approach. Staff also felt that they were not trained in palliative care; however, they recognised that it would not be that beneficial}

1Department of Psychological Medicine, 2Department of Community Nursing, Changi General Hospital, Singapore

Correspondence: Dr Tan Lay Ling, Senior Consultant, Department of Psychological Medicine, Changi General Hospital, 2 Simei Street 3 , Singapore 529889. tan.lay.ling@singhealth.com.sg 
for the PWD to be handed over to another team that the PWD and family caregivers had not built much rapport with.

Doctor Staff (DS) 4: "I don't see myself providing palliative care... to PWD or their loved ones when they are not suffering from physical symptoms... like intractable pain or dyspnoea, even though they may be in their severe stage of their dementia... and are totally dependent in their activities of daily living."

\section{Uncertainty regarding ACP and EOL care discussions}

Staff found it particularly challenging to initiate conversations regarding ACP with PWD in the earlier stages of their illness. Staff were apprehensive about the readiness of the family caregivers of PWD at a moderate to advanced phase of illness regarding care discussions. Staff also shared that the lack of a clear workflow hindered the initiation or continuation of such discussions.

NS 3: "Some family members were not keen for us to discuss such care matters either with them or the PWD. It is usually a hit and miss situation. I am not sure whether they are ready for these hard conversations. If they are ready, I find that I may not be able to systematically discuss these with them because there is no structure or clear work process."

\section{Lack of community healthcare resources to support palliative care in dementia}

Staff found it hard to coordinate the palliative care of PWD in the community. Home hospice referral was not easy when prognostication was not within six months. Limitations in CPGP funding meant that there was a lack of after-office-hour care consultations. Hence, even when ACP discussions included avoidance of unnecessary hospitalisations, family members often found it difficult to decide whether the PWD required emergency room consultations for symptoms such as fever, drowsiness and increase in agitation. Caregivers were usually reluctant to be referred to another care team, as they would have to re-establish rapport with other community healthcare providers.

NS 3: "Sometimes when the family call to ask whether they should send the PWD to the Emergency Room for complaints like fever or change in mental state, it is difficult to really advise them what to do, particularly if it is after office hours and our doctors also cannot visit to assess. It would be good to have a general practitioner who is accessible and familiar with the PWD's palliative care needs to help advise them."

\section{STRATEGIES TO ADDRESS IDENTIFIED PROBLEMS \\ Assessment of readiness of PWD and caregivers for ACP}

Team members identified the need to systematically address the lack of understanding of the progressive nature of dementia among the PWD and caregivers. A checklist (Appendix) was drafted to include readiness to engage in such difficult conversations. This checklist would be used prior to subsequent home visits to serve as a reminder to the team members regarding the palliative care approach to dementia.
DS 5: "We usually are not sure how ready they are to engage in ACP. Some of them become more convinced that the $P W D$ is becoming more deteriorated over the years. We may not always remember to check on their understanding of dementia as incurable and that they are more prepared for ACP engagement. The checklist will help to remind us how far we are with this."

\section{Staff training in ACP}

Although all team members had attended the ACP Facilitation Course, there was a perceived lack of confidence in facilitating ACP. Staff highlighted the common misperception that PWDs are unable to participate in ACP because they lack the mental capacity to do so. There was also the unfortunate deferment of Preferred Plan of Care (PPC) discussion during the early stages of illness.

NS 4: "I think ACP discussions with PWDs are more challenging because you are not always certain that they are clear with your explanations. Being able to discuss these challenges during the ACP course with experienced facilitators will be helpful."

\section{Addressing staff competencies in palliative care needs in dementia}

Although some staff had received previous training in general palliative care, most were not confident about delivering such care in the community. Departmental funding was secured for palliative care courses and conferences. Staff were able to share their learning experiences on how palliative care practices could be improved.

DS 4: "It will be good to go for further training... in palliative care to brush up on my knowledge so that I can be more confident in rendering a good standard of care..."

\section{ANALYSIS ON THE EFFECTS OF ACTION PLANS \\ Utility of checklist}

The checklist, renamed 'Facilitation of Care Planning Form' (FOCF) to better reflect its purpose, was piloted on five clients. However, although all five family caregivers endorsed that their loved ones were suffering from a terminal illness, only one proceeded to complete the ACP with PPC.

NS 2: "Using the Form... helped me have a better sense of the caregiver's understanding of dementia and I am more ready to introduce ACP to her."

\section{Reflections on ACP discussions}

Staff were more confident regarding discussions of care preferences. Although many caregivers were still unprepared to discuss ACP, team members were able to better appreciate the patients' difficulties and be supportive in their grieving process. They were also better prepared to respond to questions regarding care preferences when the caregivers were ready.

DS 6: "I find that I am more patient with the caregivers. Helping them come to terms with what is going on is more important than pushing care decisions on them." 


\section{Application of palliative care knowledge}

Although both nursing and medical staff appreciated the opportunities to attend refresher courses, the availability of palliative specialists for consultation was considered even more educational and impactful.

DS 5: "It has been really helpful attending courses and reading up on materials to keep myself abreast with palliative medicine developments but... the application of theory on the context of our patient pool is still not easy and we will need more guidance on the ground."

\section{DISCUSSION}

To the best of our knowledge, this is the first local study that investigated the adoption of palliative care principles in PWD by employing a qualitative approach. Despite mounting evidence that palliative care principles are appropriate for PWD, they are often not applied.(6) PWD are still exposed to onerous interventions that offer minimal or no benefit.

This qualitative study identified the challenges encountered by the CPGP that were also cited by previous studies. It has been reported that both professional ${ }^{(3)}$ and family carers $^{(4)}$ did not view dementia as a terminal illness. This might have contributed to the neglect in discussing EOL care for PWD and their caregivers. ${ }^{(15,16)}$ A previous study also highlighted that PWD and their families were rarely informed that dementia is a terminal illness and palliative care principles were not discussed with them. ${ }^{(4)}$

Using action research methodology, staff members were able to reflect on and critique their practice. It facilitated an examination of assumptions and habitual actions that constituted clinical practice ${ }^{(12)}$ and eventually led to an endorsement that dementia is a terminal illness regardless of the stage of severity. Team members were better able to support PWD and their family caregivers with the psychological space to come to terms with the terminal illness concept and appreciate the value of a palliative care approach. Strategies were developed by the team members themselves to systematically assess the readiness of PWD and their family carers to engage in ACP discussions using the FOCF. Staff also recognised the need to enhance their confidence in facilitating ACP conversations in the dementia population. Active exchanges between CPGP staff and ACP course facilitators clarified misperceptions regarding the inability of PWD to engage in general ACP discussions in the early stage of their illness.

Although the importance of skill enhancement in palliative care principles in key providers of dementia care has been recognised, there is a dearth of literature on educational programmes and models of care with good educational impact on improving the quality of care of patients with advanced dementia. ${ }^{(15)}$ A review of educational initiatives to improve palliative care for PWD reported that there have been few published evaluations of educational programmes, and even in studies wherein the outcomes were evaluated, the benefits for patients were not demonstrated. ${ }^{(16)}$ This qualitative study similarly highlighted the challenges of application of palliative care knowledge following training courses and conferences. The participatory action research methodology adopted was intended to enhance the quality of education of healthcare professionals in dementia care. ${ }^{(17)}$ Schemes to improve the quality of learning should be derived from a paradigm that embraces change, which is one of the characteristics of the action research methodology. The participation in action learning helped to identify problems encountered on the ground and generated solutions to equip the CPGP in their palliative care skills. The process was collaborative, flexible and circular, and allowed action with change and improvement simultaneously with research and evaluation. ${ }^{(14)}$ As the strategies were formulated by the staff themselves, they were better accepted and adopted.

A recent review of engagement of primary stakeholders in decision-making for the future care of PWD in the community highlighted the complexity of the context in which PWD, family caregivers and healthcare professionals found themselves with regard to decision-making about future care. ${ }^{(18)}$ This study was able to identify some facilitators and barriers to interdisciplinary communication between dementia care providers and palliative care in the community. Through participatory action research, CPGP members were empowered to link up with palliative specialist colleagues for consultations specific to dementia palliative care. This process facilitated continual professional development of staff and enhanced the quality of care of PWD and their family caregivers.

This study has some limitations. As it involved a community team of healthcare professionals caring for PWD and their caregivers, the insights and results shared might not be transferrable to other service settings and contexts.

In conclusion, our findings suggested that the participatory action research methodology was effective for developing strategies to facilitate staff training and engagement in palliative care provision in the community.

\section{SUPPLEMENTARY MATERIAL}

The Appendix is available online at https://doi.org/10.11622/ smedj.2021064.

\section{REFERENCES}

1. Prince MJ, Wimo A, Guerchet, M, et al; Alzheimer's Disease International. World Alzheimer Report 2015. The global impact of dementia: an analysis of prevalence, incidence, cost and trends. Available at: https://www.alz.co.uk/ research/world-report-2015. Accessed January 18, 2018.

2. Kristjanson LJ, Toye C, Dawson S. New dimensions in palliative care: a palliative approach to neurodegenerative diseases and final illness in older people. Med J Aust 2003; 179(S6):S41-3.

3. McCarthy M, Addington-Hall J, Altmann D. The experience of dying with dementia: a retrospective study. Int J Geriatr Psychiatry 1997; 12:404-9.

4. Livingston G, Sommerlad A, Orgeta V, et al. Dementia prevention, intervention, and care. Lancet 2017; 390:2673-734.

5. Volicer L, Simard J. Palliative care and quality of life for people with dementia: medical and psychosocial interventions. Int Psychogeriatr 2015; 27:1623-34.

6. Subramaniam M, Chong SA, Vaingankar JA, et al. Prevalence of dementia in people aged 60 years and above: results from the WiSE study. J Alzheimers Dis 2015; 45:1127-38.

7. Tew CW, Tan LF, Luo N, Ng WY, Yap P. Why family caregivers choose to institutionalize a loved one with dementia: a Singapore perspective. Dement Geriatr Cogn Disord 2010; 30:509-16.

8. Vaingankar JA, Subramaniam M, Picco L, et al. Perceived unmet needs of informal caregivers of people with dementia in Singapore. Int Psychogeriatr 2013; 25:1605-19.

9. Lien Centre for Palliative Care, Singapore; Duke-NUS Medical School, Singapore. Report on the national strategy for palliative care. Available at: https:// singaporehospice.org.sg/site2019/wp-content/uploads/Report_on_National_ 
Strategy_for_Palliative_Care-5Jan2012.pdf. Accessed January 8, 2018.

10. Sin GL, Ng LL. The Community Psychogeriatric Programme: a pilot project in the eastern sector of Singapore. Singapore Med J 2011; 52:463-5.

11. Lo TJ, Neo PS, Peh TY, et al. Improving quality of palliative care through implementation of national guidelines for palliative care. J Palliat Med 2019; 22:1439-44.

12. Kemmis S. Action research as a practice-based practice. Educ Action Res 2009; $17: 463-74$

13. Street A, Robinson A. Advanced clinical roles: investigating dilemmas and changing practice through action research. J Clin Nurs 1995; 4:349-57.

14. Kemmis S, McTaggart R, Nixon R. The Action Research Planner: Doing Critical Participatory Action Research. Singapore: Springer, 2014.
15. Harrison Dening K, King M, Jones L, Vickerstaff V, Sampson EL. Advance care planning in dementia: do family carers know the treatment preferences of people with early dementia? PLoS One 2016; 11 :e0159056.

16. Sampson EL, Gould V, Lee D, Blanchard MR. Differences in care received by patients with and without dementia who died during acute hospital admission: a retrospective case note study. Age Ageing 2006; 35:187-9.

17. Carter G, van der Steen JT, Galway K, Brazil K. General practitioners' perceptions of the barriers and solutions to good-quality palliative care in dementia. Dementia (London) 2017; 16:79-95.

18. Chang E, Hancock K, Harrison K, et al. Palliative care for end-stage dementia: a discussion of the implications for education of health care professionals. Nurse Educ Today 2005; 25:326-32. 

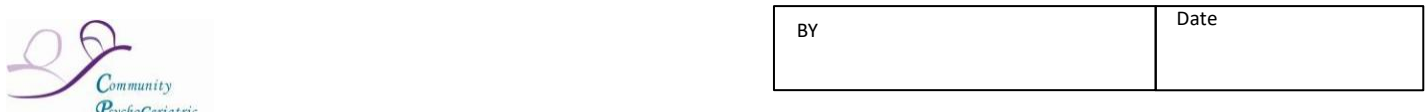

\section{Progarame Facilitation of Care Planning}

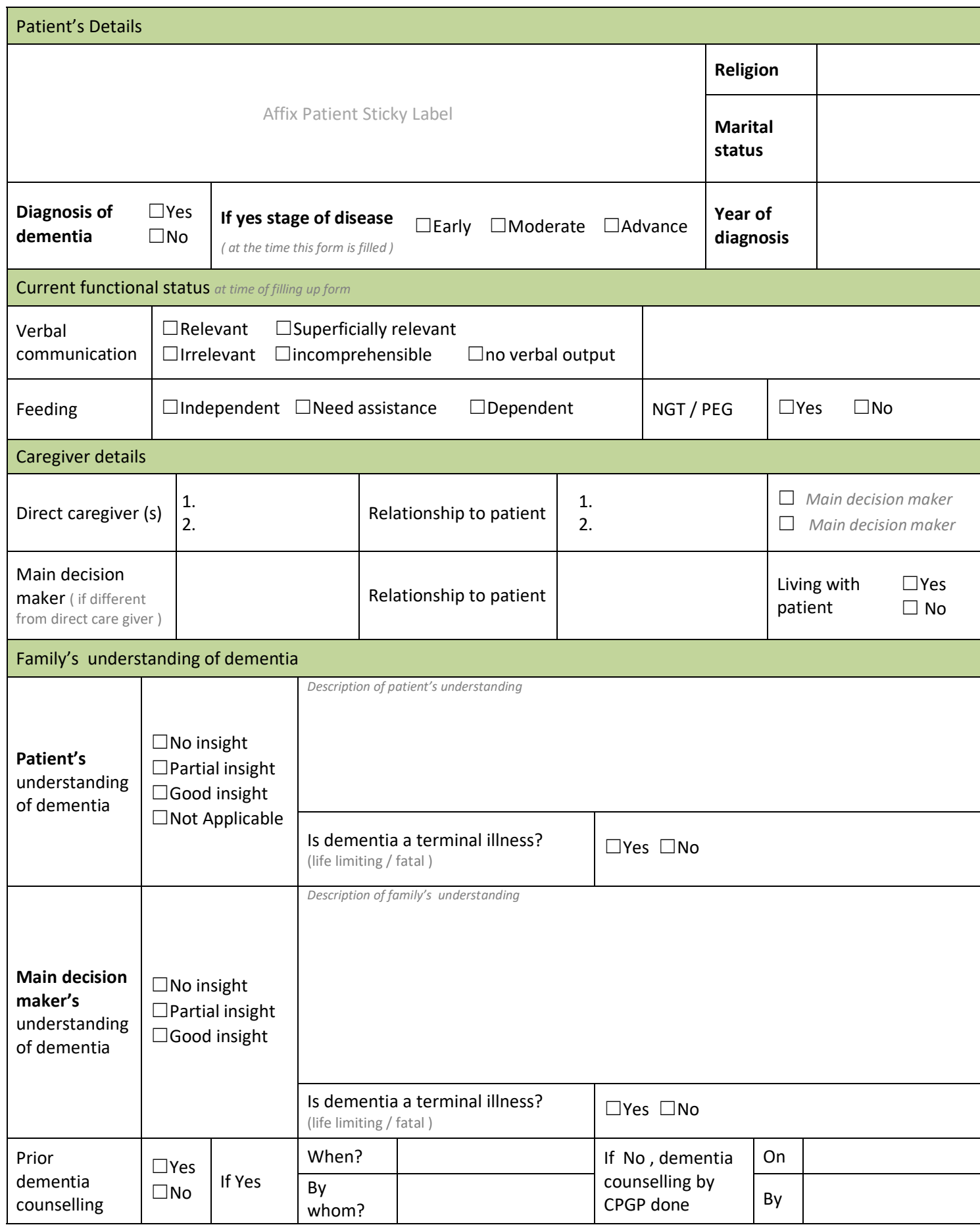

$\sim 1 \sim$ 


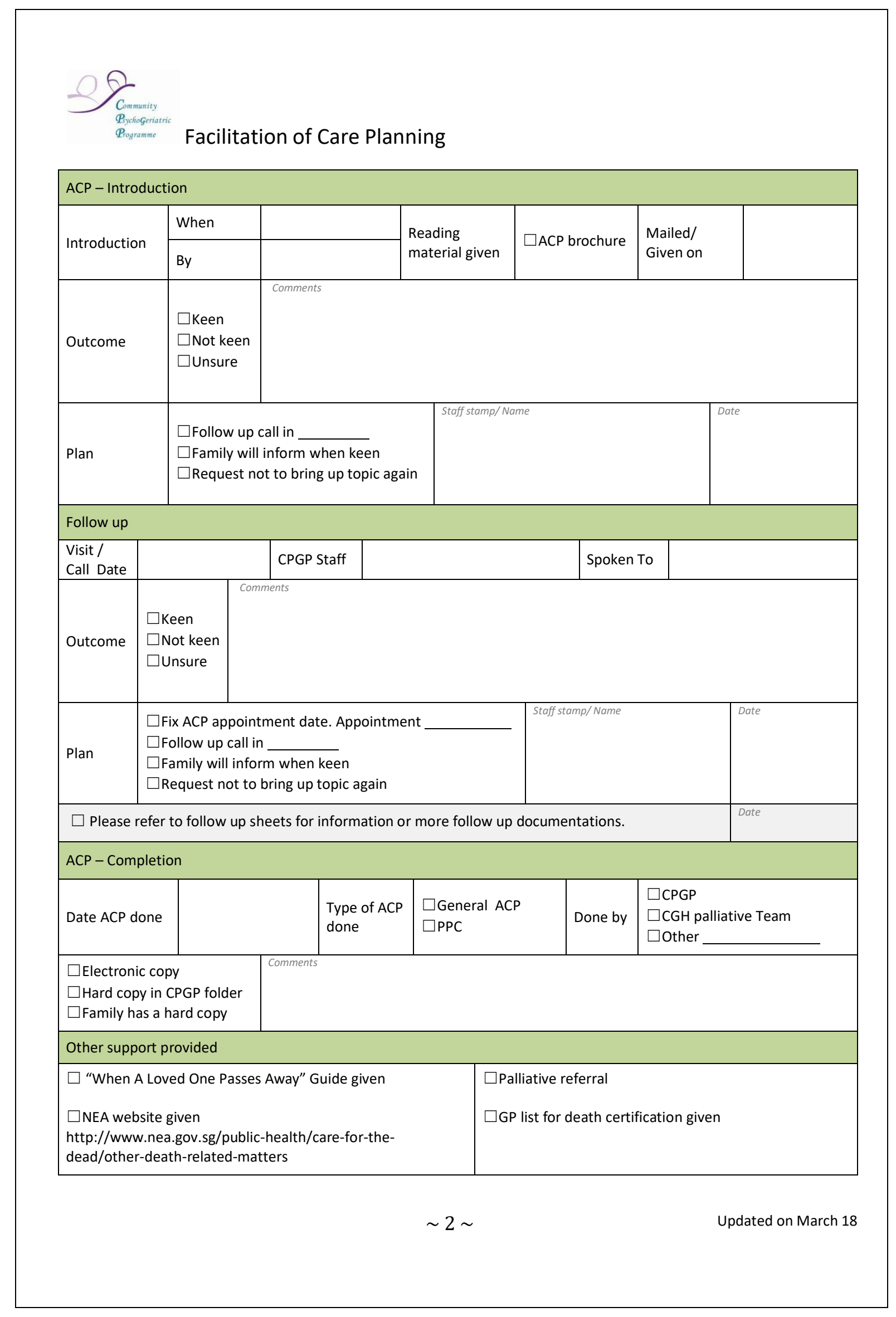

